\title{
Morbidity and Mortality Review in a University Dental Hospital: A Necessary Tool to Improve Quality of Care
}

\author{
Rémi Esclassan 1,2,3,๑ Marie-Cécile Valera ${ }^{1,2,4}$ Jean Marc Bergia ${ }^{2}$ Thibault Canceill ${ }^{1,2,5}$ \\ Leonor Costa Mendes ${ }^{1,2}$ Isabelle Bailleul-Forestier ${ }^{1,2}$ Virginie Gardette ${ }^{2,6}$ Frédéric Vaysse ${ }^{1,2,3}$ \\ Marie Gurgel-Georgelin ${ }^{1,2}$ Emmanuelle Noirrit ${ }^{1,2,4}$
}

${ }^{1}$ Faculté de Chirurgie Dentaire de Toulouse, 03 chemin des

Maraîchers, Toulouse, France

${ }^{2} \mathrm{CHU}$ Rangueil, 03 chemin des Maraîchers, Toulouse, France

${ }^{3}$ Laboratoire AMIS, CNRS, Toulouse, France

${ }^{4}$ I2MC, CHU de Toulouse and Université de Toulouse, Toulouse,

France

${ }^{5}$ CIRIMAT, University of Toulouse, CNRS, INPT, Université Paul

Sabatier, Faculté de Pharmacie, Toulouse, France

6 UMR INSERM, Université Paul Sabatier, CHU de Toulouse,

Hôtel-Dieu, Toulouse, France

Address for correspondence Rémi Esclassan, PhD, DDS, Faculté de Chirurgie Dentaire de Toulouse, 03 chemin des Maraîchers, Toulouse 31059, France (e-mail: esclassan.r@gmail.com).

Eur J Dent 2021;15:307-311

\begin{abstract}
Keywords

- mortality and morbidity review

- dental university hospital

- midazolam anaphylaxis

- tooth inhalation

- perforation

- inmate
\end{abstract}

Objective The study aimed to describe and to analyze the first morbidity and mortality review (MMRs) set up within a Dental University Hospital using detailed case reports to highlight the benefits of MMRs for patients, practitioners, teachers and to implement appropriate protocols to prevent recurrence.

Materials and Methods The MMRs were performed within the dentistry departments of the hospital over the 1-year study period. Each case was reviewed according to a protocol based on a tool defined by the Clinical Risk Unit and the Association of Litigation and Risk Management (ALARM).

Results Four cases were selected based on an oral report by a doctor from the dental service, a downstream service, or by the attending physician. The first case report related to a patient who suffered a breathing shock. The second concerned a tooth inhalation by a young disabled boy. The third was a therapeutic failure instigated by a student during a tooth preparation, and the fourth case involved an unexpected face-to-face meeting between a prisoner accompanied by police guards and an ancient victim at the dental hospital.

Discussion Clinical incidents were investigated with the ALARM protocol. This process is also less focused on the individual who makes the error and more on contributing systemic factors. The systematic analysis of cases associated with bibliographic reviews improves learning and performance outcomes. Clear answers were given in response to the problems raised during these MMRs.

Conclusion In dental hospitals, the culture of MMRs needs to be integrated into resident training like in medical hospitals. published online

January 7, 2021
DOI https://doi.org/

10.1055/s-0040-1719233

ISSN 1305-7456.
(C) 2021. European Journal of Dentistry.

This is an open access article published by Thieme under the terms of the Creative Commons Attribution License, permitting unrestricted use, distribution, and reproduction so long as the original work is properly cited. (https:// creativecommons.org/licenses/by/4.0/)

Thieme Medical and Scientific Publishers Pvt. Ltd., A-12, 2nd Floor, Sector 2, Noida-201301 UP, India 


\section{Introduction}

A morbidity and mortality review (MMR) is a collective review of the medical files of a patient whose outcome has been marked by an adverse event such as death or the occurrence of a complication. It consists in examining whether the patient's management was appropriate and identifying potential failures that may have contributed to the development of the complication. ${ }^{1}$ This concept was first implemented in the United States at the beginning of the 20th century as part of the initial training of surgeons. ${ }^{2}$ The aim was to learn from cases where death or malfunction had occurred to improve professional practices by analyzing in a collegial manner the occurrence of deaths unprejudiced by any individual judgment or sanctions. ${ }^{3}$ The concept then spread to other disciplines and other countries. ${ }^{4}$ The emergence of quality management and risk management in hospitals in the 1990s changed the way we looked at MMRs; the latter subsequently emerged as a tool for improving the quality of care and management of iatrogenic risks and were deemed particularly useful for students. ${ }^{5}$ MMRs are currently routinely implemented in different medical services such as surgery, anesthesia resuscitation, oncology, and internal medicine.

To the best of our knowledge, the current literature however does not include any MMR experiences from dental hospitals. The objectives of the current study are to describe and analyze the first MMRs set up within a Dental University Hospital using detailed case reports to highlight the benefits of MMRs for patients, practitioners, teachers, and interns and to implement appropriate protocols to prevent recurrence.

\section{Materials and Methods}

We conducted a retrospective analysis of the first dental unit patient medical records archived as part of the larger university hospital MMRs. The dental services appointed one MMR manager to operate under the auspices of the head of the dental unit and in accordance with a drafted charter in compliance with the high autority of health (HAS) recommendations. Cases were selected based on an oral report by a doctor from the dental service, a downstream service, or by the attending physician. No severity or frequency levels were required. Any adverse events or unintended outcomes could be reported by practitioners, students, nurses, or nurse managers by way of a standardized digital form. In our hospital, the appointed driver of MMRs is the nurse manager, who had been trained in MMR procedures as part of his previous position in oncology. The MMR secretary is the medical director. Four MMRs (one every 3 months) were performed within the dentistry departments of Toulouse Dental Hospital over the 1-year study period. Meetings were held at lunch time and one case report was reviewed per meeting that took approximately 1 hour (-Table 1 ).

Each case was reviewed according to a structured and systematic protocol based on a tool defined by the Clinical Risk Unit and the Association of Litigation and Risk Management (ALARM). ${ }^{6}$ This tool was initially derived from research in the
Table 1 Summary table of the sequential phases of morbidity and mortality reviews

\begin{tabular}{l}
\hline Expansion of the attendance list \\
\hline $\begin{array}{l}\text { Reminder of the MMR values, the right to free expression which } \\
\text { applies to everyone, the assurance of anonymity both for the } \\
\text { patient and the practitioner }\end{array}$ \\
\hline $\begin{array}{l}\text { PowerPoint presentation of case files by the rapporteur(s), paying } \\
\text { particular attention to respect the anonymity of patients as well } \\
\text { as professionals }\end{array}$ \\
\hline $\begin{array}{l}\text { Evaluation of the criticality of the event (hospital scale) and its } \\
\text { potential frequency }\end{array}$ \\
\hline $\begin{array}{l}\text { Identification of problems encountered by the team and alterna- } \\
\text { tives for dealing with them }\end{array}$ \\
\hline $\begin{array}{l}\text { In-depth search for causes using the systemic analysis method } \\
\text { (ALARM grid) }\end{array}$ \\
\hline Collective discussion on the lessons to be drawn from the analysis \\
\hline $\begin{array}{l}\text { The proposal and collective definition of actions required for } \\
\text { improvement }\end{array}$ \\
\hline Follow-up of actions defined in previous MMRs \\
\hline
\end{tabular}

Abbreviations: ALARM, Association of Litigation and Risk Management; MMR, morbidity and mortality review.

aviation and nuclear industries, and provides a framework for the systemic analysis of the causes of any adverse events. It explores institutional contexts, organizational and management factors, work environment factors, individual factors, team factors, and patient factors. ${ }^{6}$

The referent practitioner in charge of MMRs reviewed files presented during 2017 to 2018 to analyze their content. The minutes of each conference provided information regarding the number of participants attending individual meetings, the patient's gender and sex, the theme and reason for admission to the dental hospital, the circumstances of the adverse event, and the defined action plan.

We collected orally the patient's nonopposition for the publication of anonymized case reports. This article has complied with all relevant federal guidelines and institutional policies.

\section{Description of Case Reports Selected}

An average of 12 participants, representing each dental specialty, attended to the four MMRs.

The first case report related to a patient who suffered a breathing shock, which occurred during a conservative dental intervention under conscious sedation. The patient was a young teenage boy (16 and a half years of age) with multiple malformations combining psychomotor retardation, gastroesophageal reflux with merycism, and food allergies. He had been followed up in the service since march 2006 and had previously undergone procedures requiring general anesthesia as well as midazolam and nitrous oxide. About 15 minutes after administration of midazolam intra rectal (IR) (10 mg) and at the end of the administration of local anesthesia $(0.9 \mathrm{~mL}$ of articaine $1 / 200,000$ intraosseous injected with the Quicksleeper), he developed respiratory distress without swelling or rash. The patient was immediately placed in an upright sitting position and administered oxygen from a wall 
outlet. The patient was monitored with a pulse oximeter. Because oxygen saturation decreased to $89 \%$, the practitioner requested direct assistance from the anaesthetist who was consulting nearby. The clinical observation reported a hemodynamic failure, tachycardia, impregnable tension, bradypnea, $84 \%$ saturation in ambient air, and cold extremities. No mottling, no cyanosis, and no sweating were diagnosed at the time. Anaphylaxis is an acute life-threatening hypersensitivity reaction following re-exposure to a sensitized antigen. Drug-induced anaphylaxis and the male gender are factors that increase the risk of severe anaphylactic shock. ${ }^{7}$ Clinical manifestations include skin symptoms followed by cardiovascular, respiratory, gastrointestinal, and neurologic symptoms. However, our patient did not develop any skin manifestations. The consultation ward was not equipped with an emergency cart, and no oxygen cylinder was available for transferring the patient to the emergency ward.

Finally, an emergency practitioner injected adrenaline (Epipen-epinephrine auto injector-0.3mg IM). The patient was transferred to the emergency room, where he received the appropriate treatment (dexchlorpheniramine 1 ampoule, methylprednisolone $50 \mathrm{mg}$ IVD, albuterol sulfate inhalation aerosol, subcutaneous injection of $1 / 2$ vial of terbutaline; high-flow $\mathrm{O}_{2}$ mask at $8 \mathrm{~L} / \mathrm{min}$ ). He was monitored for a 24-hour period. Several weeks later, test results confirmed an allergy to midazolam (but not to the local anesthesia). During the MMR, we discussed the failure of the dentist to diagnose anaphylactic shock, the long lag time (around 10 minutes) required to find the appropriate equipment (emergency cart with high-flow $\mathrm{O}_{2}$ mask) and medication (adrenalin, IV solution, oxygen bottle to move the patient to the emergency ward) that resulted in delaying management of the patient and insertion of the venous line by a consultation service at the hospital.

This adverse event led to the implementation of several corrective measures: the dentist's understanding of emergency procedures was updated; a verified and fully stocked emergency cart is now always available and new emergency number displays were installed.

The second case report concerned a tooth inhalation, by a young 4-year-old disabled (Down syndrome) boy. ${ }^{8}$ After an injury resulting in a complex coronal fracture with pulp exposure of the 61 (upper left deciduous incisor), the tooth was extracted under local anesthesia. Despite using an equimolar oxygen nitrous oxide mix, the patient became very restless. Due to the sudden head movement of the child just after the extraction, the tooth slipped out of the forceps, fell on the tongue, and disappeared into the pharynx. As the young boy did not exhibit any obvious signs of respiratory distress and no cough response was elicited, it was assumed that the patient had swallowed the tooth. At the time of tooth avulsion, the mother fainted and the dental nurses took care of her. The young boy was playing at the time of his mother's recovery.

Both the patient and his mother were subsequently discharged with supervisory advice. Total 2 months later, the patient was admitted to the emergency department because of a sustained recurrent cough and fever that had lasted for several days and failed to respond to three different antibiotic therapies. The cardiopulmonary auscultation was normal. A chest X-ray confirmed the presence of a foreign body inside the left lung. It had caused an infection in the left lower pulmonary lobe, which was treated with antibiotics (clavulanic acid and penicillin) and anti-inflammatories (prednisolone), prior to surgery. Two endoscopies, under general anesthesia, were required to recover the tooth. The 1-year follow-up indicated no further signs of lung infection.

The third case report was a therapeutic failure instigated by a student during a tooth preparation. The 75-year-old patient was treated in the dentistry department to construct two maxillary posterior bridges to replace two missing teeth (25 and 16). The student made a perforation while preparing a root canal for a post and core on tooth 24 , which was supposed to be a bridge abutment. He reached the periodontal ligament of 23 with the drill. The teaching team set the drilling indication. The student sought help when he realized that the post was completely blocked in the root and inaccessible. Fortunately, after treatment with mineral trioxide aggregate, teeth 23 and 24 were kept on the arch but became useless as bridge abutment. Using the ALARM protocol, we identified the presence of an unusually large number of supervisors among other organizational and management factors that contributed to the failure. A first student performed endodontic treatment of the tooth. But preparation for the core inlay took place 7 months later and was performed by a second student (in his 5th year of dentistry). Regarding individual factors, the second student had previously performed a similar preparation on another tooth, but this particular day, he did not work with his normal partner (team factor).

Finally, the fourth case involved an unexpected face-to-face meeting between a prisoner accompanied by police guards and an ancient victim at the Dental Hospital. The two individuals had surgery scheduled at the same time in two adjacent operating rooms. This unfortunate meeting in the corridor was a big shock to the victim who reported a traumatic revivification episode. ${ }^{9}$

\section{Discussion}

The morbidity and mortality conference is arguably one of our most powerful teaching tools and according to Indresano ${ }^{10}$, "the objectives of the conference are to learn from complications and errors, modify behavior, and judgment based on previous experience and prevent the repetition of errors leading to complications."

The reported cases led to a bibliographical analysis. According to the Japanese Dental Society, between 19 and $44 \%$ of dentists encounter a medical emergency in any single calendar year. ${ }^{11}$ In the United States and Canada, a syncope was the most common medical emergency seen by dentists, representing around $50 \%$ of all emergencies followed by mild allergies, representing $8 \%$ of all emergencies. Other emergencies have also been reported such as angina pectoris/ myocardial infarction, cardiac arrest, postural hypotension, seizures, bronchospasm, and diabetic emergencies. ${ }^{12}$ In fact, rash or urticaria is less common in drug-induced than 
food-induced anaphylaxis. ${ }^{7}$ Midazolam is widely used for its anxiolytic and sedative properties. Midazolam anaphylaxis only occurs rarely (1.4\% according $\mathrm{Kim}^{7}$ ) and is sparingly described in the literature. ${ }^{13,14}$ The current study highlights that rare adverse reactions such as anaphylactic shock do occasionally occur and that emergency equipment should be available everywhere in the hospital, even in the consultation wards. Moreover, special needs patients should be carefully monitored because they often have confounding medical disabilities and because they have difficulties expressing themselves especially when something is wrong.

The second MMR analyzed a pulmonary complication related to a tooth inhalation. This kind of event is not frequently described, although there is much literature about foreign body inhalation and ingestion..$^{15}$ Foreign substances are ingested $87 \%$ of the time versus aspirated. ${ }^{16}$ Tiwana's review ${ }^{15}$ found 36 reports of aspiration or ingestion over a 10-year time period, but they all concerned dental materials, not teeth. Aspirated or ingested dental foreign objects included dental implant screwdrivers, burs, crowns, orthodontic wire, and implants. Choking due to foreign body aspiration is the leading cause of death in children between 0 and 2 years of age and plays a considerable role up to the age of 14 years. ${ }^{17}$ As was the case in our patient, the therapy of choice in these events is retrieval of the aspirated foreign body by endoscopy (laryngoscopy and bronchoscopy). This procedure always requires a general anesthesia and subsequent hospitalization, particularly when young patients are affected. It is never without risks and unfortunately in some cases even after successful retrieval; death can occur during the recovery phase. ${ }^{16}$ It is also noteworthy that according to the parents of this particular patient, two dentists and one small city hospital center did not wish to take care of their son because of his disability. Conscious sedation for dental care is not very common outside of the hospital setting in France. Moreover, disabled patients are often referred to a hospital because they require longer treatment times. That could explain why two of our reports of adverse events involved this type of patient profile. Practitioners should keep in mind that disabled patients are at greater risk of complications, and that chest radiography is recommended in any cases where there is suspicion that a foreign body has been ingested or inhaled, whether the patient is disabled or not.

The third case related a therapeutic mistake during a tooth preparation for a post and core, abutment of a dental bridge. Raustia et $\mathrm{al}^{18}$ noted that the most frequent complication and failure related to fixed metal ceramic bridge prostheses and perpetrated by dental students occurred during pre-prosthetic endodontic treatment of abutment teeth and during preparation of the root canals for a cast core. This can be explained by the dental student's lack of experience. ${ }^{18}$ Good practices in prosthetics as in endodontics are taught but need to be rehearsed before the actual intervention. ${ }^{19,20}$

The fourth case report is unfortunate. The fact that a young patient who was a victim of a prior aggression could meet her aggressor in the same dental hospital because their appointments coincided is quite a rare event, and to the best of our knowledge, no similar case has been previously reported in literature. This situation occurred because the operating rooms are located in a specific area of the clinic at the end of a corridor. Normally, inmate patients are treated in the prison except for specific procedures such as oral surgery or specialized radiography. In these cases, they are referred to the dental hospital. The inmates' access and passage through the hospital has now been modified to avoid any visual contact with the other patients.

We investigated clinical incidents with the ALARM protocol which consists of a series of steps that facilitate the generation of a formal report. ${ }^{6}$ This grid, adapted to health care institutions, classifies all causes into categories allowing a systematic exploration of the context of the event from a sphere close to the act of care (patient, tasks to be performed, and caregiver), to organizational layers that are increasingly distant (team, work environment, organization and management, and institutional context).

This process is also less focused on the individual who makes the error and more on contributing systemic factors. MMRs should not blame health care professionals but rather improve health care quality and patient safety, and provide support for reporting incidences. ${ }^{21}$ Furthermore, MMRs provide teaching opportunities for trainees as well as clinicians: the systematic analysis of cases associated with bibliographic reviews improves learning and performance outcomes. $^{22}$

After a 1-year implementation period in our dental hospital, the concept was well understood and accepted by

Table 2 Summary of the problems encountered and the corrective actions implemented after the mortality and morbidity conference

\begin{tabular}{|l|l|}
\hline Problems & Decisions \\
\hline $\begin{array}{l}\text { Breathing } \\
\text { shock } \\
\text { coning the }\end{array}$ & $\begin{array}{l}\text { Available monitoring during treatment sessions } \\
\text { with midazolam IR injection } \\
\text { Provision of a verified and fully stocked emer- } \\
\text { gency cart } \\
\text { Clear display of emergency numbers } \\
\text { Mandatory and continuous clinical monitoring } \\
\text { per and postoperatively } \\
\text { Refresher emergency skills training for dental } \\
\text { practitioner }\end{array}$ \\
\hline $\begin{array}{l}\text { Tooth } \\
\text { inhalation }\end{array}$ & $\begin{array}{l}\text { Systematically prescribing a chest X-ray when a } \\
\text { foreign body disappears in the oropharynx }\end{array}$ \\
\hline $\begin{array}{l}\text { Mistake } \\
\text { puring tooth }\end{array}$ & $\begin{array}{l}\text { The student who drills the root canal is the one } \\
\text { who makes the root treatment } \\
\text { X-ray check after gates drill n³ } \\
\text { Close supervision of any “risky act" performed } \\
\text { for the first time by the student, even a final } \\
\text { year student } \\
\text { Working in student pairs: clinical acts such } \\
\text { as root preparation should be done with the } \\
\text { presence of an assistant }\end{array}$ \\
\hline $\begin{array}{l}\text { Unfortunate } \\
\text { "meeting" in } \\
\text { the wait- } \\
\text { ing room }\end{array}$ & $\begin{array}{l}\text { Changing the inmates' passage through the } \\
\text { dental hospital to limit contact with the public } \\
\text { (installation of an external access doorbell, } \\
\text { privacy management) } \\
\text { Communication of the procedure with the } \\
\text { prison administration, medical, and paramedi- } \\
\text { cal care teams. }\end{array}$ \\
\hline
\end{tabular}


hospital practitioners. Attitudes toward MMRs, particularly among residents, were generally positive. Clear answers were given in response to the problems raised during these MMRs and specific protocols were designed to overcome individual problems (-Table 2 ).

In dental hospitals, the "culture" of MMRs needs to be integrated into resident training like it is the case in medical hospitals. The next step will be to involve more and more clinicians to attend these conferences.

\section{Conflict of Interest}

None declared.

\section{References}

1 Vallé B, Gasq C, Dehours E, et al. Morbidity and mortality conferences in emergency departments: the French National Survey. Eur J Emerg Med 2013;20(5):364-366

2 Orlander JD, Barber TW, Fincke BG. The morbidity and mortality conference: the delicate nature of learning from error. Acad Med 2002;77(10):1001-1006

3 Rodziewicz TL, Hipskind JE, Medical error prevention. StatPearls, Treasure Island, FL: StatPearls Publishing; 2019

4 Ferreira KS, Lynch K, Ryder BA, Connolly M, Miner T, Harrington DT. Lessons learned from the surgical morbidity and mortality conference. J Surg Educ 2019;76(1):174-181

5 Sacks GD, Lawson EH, Tillou A, Hines OJ. Morbidity and mortality conference 2.0. Ann Surg 2015;262(2):228-229

6 Vincent C, Taylor-Adams S, Chapman EJ, et al. How to investigate and analyse clinical incidents: clinical risk unit and association of litigation and risk management protocol. BMJ 2000;320(7237) :777-781

7 Kim S-Y, Kim M-H, Cho Y-J. Different clinical features of anaphylaxis according to cause and risk factors for severe reactions. Allergol Int 2018;67(1):96-102

8 Canceill T, Esclassan R, Marty M, Valera MC, TrzaskawkaMoulis E, Noirrit-Esclassan E. Misdiagnosed tooth aspiration in a young handicapped boy: case report and recommendations. Case Rep Dent 2019;2019:8495739
9 Disdier P. [The post-traumatic nightmare or the ghost of past trauma]. Soins Psychiatr 2017;38(310):41-44

10 Indresano AT. The morbidity and mortality conference: a case assessment tool, quality control measure, and teaching method. J Am Coll Dent 2004;71(3):23-25

11 Matsuura H. Analysis of systemic complications and deaths during dental treatment in Japan. Anesth Prog 1989;36(4-5): 223-225

12 Haas DA. Management of medical emergencies in the dental office: conditions in each country, the extent of treatment by the dentist. Anesth Prog 2006;53(1):20-24

13 Landsem LM, Ross FJ, Eisses MJ. A case of midazolam anaphylaxis during a pediatric patient's first anesthetic. J Clin Anesth 2017;43:75-76

14 Jeon YS, Shim J, Jun EH, Choi ST, Jung HS. Midazolam anaphylaxis during general anesthesia: a case report. Medicine (Baltimore) 2019;98(41):e17405

15 Tiwana KK, Morton T, Tiwana PS. Aspiration and ingestion in dental practice: a 10-year institutional review. J Am Dent Assoc 2004;135(9):1287-1291

16 Kim E, Noh W, Panchal N. Mortality from an aspiration of dental crown during extraction. Gerodontology 2017;34(4):498-500

17 Göktas O, Snidero S, Jahnke V, Passali D, Gregori D. Foreign body aspiration in children: field report of a German hospital. Pediatr Int 2010;52(1):100-103

18 Raustia AM, Näpänkangas R, Salonen AM. Complications and primary failures related to fixed metal ceramic bridge prostheses made by dental students. JOral Rehabil 1998;25(9):677-680

19 Goodacre CJ, Spolnik KJ. The prosthodontic management of endodontically treated teeth: a literature review. Part III. Tooth preparation considerations. J Prosthodont 1995;4(2):122-128

20 Alrahabi M, Zafar MS, Adanir N. Aspects of clinical malpractice in endodontics. Eur J Dent 2019;13(3):450-458

21 Cooper J, Edwards A, Williams H, et al. Nature of blame in patient safety incident reports: mixed methods analysis of a national database. Ann Fam Med 2017;15(5):455-461

22 Benassi P, MacGillivray L, Silver I, Sockalingam S. The role of morbidity and mortality rounds in medical education: a scoping review. Med Educ 2017;51(5):469-479 